\title{
Inaccuracy of glomerular filtration rate estimation from height/plasma creatinine ratio
}

\author{
R Skinner, M Cole, A D J Pearson, M J Keir, L Price, R A Wyllie, M G Coulthard, \\ A W Craft
}

\begin{abstract}
Use of a height/plasma creatinine formula to estimate glomerular filtration rate (GFR) is simpler and less invasive than renal or plasma clearance methods. The aim of this study was to determine whether these formulas enabled accurate prediction of GFR measured from the plasma clearance of ${ }^{51} \mathrm{Cr}$ labelled ethylenediaminetetra-acetic acid ( ${ }^{51}$ Cr-EDTA). Thirty nine patients underwent GFR measurement at least six months after potentially nephrotoxic chemotherapy. Altman-Bland analysis was performed on the measured GFR and that estimated simultaneously using the original and a modified Counahan-Barratt formula and the Schwartz formula. The limits of agreement of the estimated GFR with the measured GFR were unacceptably wide in each case, despite highly significant correlation coefficients. The bias was smallest for the modified Counahan-Barratt formula. Use of these formulas to estimate GFR in children is insufficiently accurate for research purposes and has limitations in clinical practice. Furthermore, use of correlation coefficients to evaluate different methods of measuring GFR is inappropriate.
\end{abstract}

(Arch Dis Child 1994; 70: 387-390)

Department of Child Health, Medical

School and the Royal

Victoria Infirmary,

Newcastle upon Tyne

R Skinner

A D J Pearson

L Price

R A Wyllie

$M$ G Coulthard

A W Craft

Departments of Child Health and of Medical Statistics, The Medical School, Newcastle upon Tyne M Cole

Department of Medical Physics, Royal Victoria Infirmary, Newcastle upon Tyne M J Keir

Correspomdence to: Dr R Skinner, Department of Child Health, The Medical School, Framlington Place, Newcastle upon Tyne NE2 $4 \mathrm{HH}$

Accepted 18 December 1993

Measurement of the glomerular filtration rate (GFR) in children and adolescents is an important part of the assessment of many renal diseases, and may facilitate safer prescribing of fluids, electrolytes, and drugs. ${ }^{1}$ It is of particular value in children with malignant disease in whom it may provide an important measure of toxicity, ${ }^{2}$ and influence the type and dose of cytotoxic drug treatment.

In paediatric practice, however, both the acceptability and accuracy of conventional renal clearance methods of GFR measurement are reduced by the need for carefully timed and complete urine collections. The utility of the inulin clearance method is limited further by the requirement for a continuous intravenous infusion, and the cumbersome nature of the inulin assay. The utility of endogenous creatinine clearance measurement is diminished by variability in the dietary intake and tubular secretion of creatinine, and by with the plasma creatinine assay. There is often considerable discrepancy between GFR values from the two techniques in individual patients, with the latter method usually giving higher results due to tubular creatinine secretion. ${ }^{3}$

These disadvantages prompted the introduction of clinical measurement of GFR from the plasma clearance of ethylenediaminetetraacetic acid labelled with ${ }^{51}$ chromium $\left({ }^{51} \mathrm{Cr}\right.$ EDTA), without the necessity for urine collection. ${ }^{4}$ Initial studies in adults ${ }^{56}$ and in children ${ }^{78}$ showed that ${ }^{51} \mathrm{Cr}$-EDTA plasma clearance corresponded closely to simultaneously measured renal inulin clearances. The simplicity, wide availability, and low radiation exposure ${ }^{5}$ of radioisotopic plasma clearance methods have led to their widespread adoption in paediatric practice.

Several attempts have been made to introduce and validate simpler methods whereby GFR can be estimated from the plasma creatinine concentration $\left(P_{c r}\right)$, assuming steady state conditions. Two well known formulas have been published for use in children, ${ }^{9} 10$ both of which allow determination of GFR from $P_{c r}$ and height. These formulas have been validated by the use of correlation coefficients to compare the measured and estimated GFR, but this method is inappropriate and does not indicate whether the estimated GFR is an acceptably accurate substitute for measured GFR. ${ }^{11}$

The aim of this study was to determine whether GFR measured from the plasma clearance of ${ }^{51} \mathrm{Cr}$-EDTA could be predicted accurately and reliably by use of a formula relating the patient's height and $P_{c r}$. interference of non-creatinine chromogens

\section{Patients and methods}

Data were collected prospectively during a study of renal function after treatment in a cohort of 39 consecutive children and adolescents ( 22 male) with malignant solid tumours completing treatment with potentially nephrotoxic chemotherapy (cisplatin (23 patients), ifosfamide (13), or both (three)) at the Paediatric Oncology Unit in Newcastle upon Tyne. All patients were investigated at least six months after completion of chemotherapy for neuroblastoma (10 patients), rhabdomyosarcoma (six), soft tissue sarcoma (five), Ewing's sarcoma (five), osteosarcoma (four), brain tumour (four), germ cell tumour (three), and hepatic tumour (two).

The patient's height was measured using a stadiometer (Raven Equipment Ltd). No patient was underweight, but four were overweight. ${ }^{12}$ No patient had clinically detectable oedema, pleural or ascitic fluid.

The ${ }^{51} \mathrm{Cr}$-EDTA plasma clearance determination of GFR was performed using a single 
intravenous injection of radioisotope at time 0 , with subsequent collection of plasma samples at 1,2 , and 4 hours. A straight line was fitted to the log activity time curve, assuming adequate distribution of the tracer by 1 hour, and the half time $\left(t_{1 / 2}\right)$ of the radioisotope determined. The volume of distribution $\left(\mathrm{V}_{\mathrm{D}}\right)$ was found from the estimate at time 0 . The plasma clearance was calculated from

$$
\text { Plasma clearance }(\mathrm{ml} / \mathrm{min})=\frac{\mathrm{V}_{\mathrm{D}} \times 0.693}{\mathrm{t}_{1 / 2}}
$$

and then expressed per $1.73 \mathrm{~m}^{2}$ surface area, giving the measured GFR.

The $P_{c r}$ of one of the above blood samples was measured by a Technicon SMAC AutoAnalyser (Jaffe method with initial dialysis). The estimated GFR was calculated in three ways:

(1) the Counahan-Barratt formula ${ }^{9}$

$$
\mathrm{GFR}=\frac{38 \times \mathrm{ht}}{\mathrm{P}_{\mathrm{cr}}}
$$

where $\mathrm{GFR}=$ glomerular filtration rate $\left(\mathrm{ml} / \mathrm{min} / 1.73 \mathrm{~m}^{2}\right), \quad \mathrm{ht}=$ height $(\mathrm{cm})$, and $\mathrm{P}_{\mathrm{cr}}=$ plasma creatinine concentration $(\mu \mathrm{mol} / \mathrm{l})$ (2) a modified Counahan-Barratt formula (Morris et al) 13

$$
\mathrm{GFR}=\frac{40 \times \mathrm{ht}}{\mathrm{P}_{\mathrm{cr}}}
$$

(3) the Schwartz formula ${ }^{10}$

$$
\mathrm{GFR}=\frac{\mathrm{k} \times \mathrm{ht}}{\mathbf{P}_{\mathrm{cr}}}
$$

where $k=48.6$ except in males $\geqslant 13$ years age, in whom $k=61.9$.

The values of $k$ (48.6 and 61.9) used in formula (3) are derived by multiplying those figures $(0.55$ and 0.70$)$ quoted by Schwartz et $a l^{1}$ by a factor of $88 \cdot 4$ to allow for expression of $P_{c r}$ in $\mu \mathrm{mol} / 1$ in this study, rather than $\mathrm{mg} / \mathrm{dl}$.

Counahan et al used the true $P_{c r}$ (either measured directly or derived by subtracting $12.4 \mu \mathrm{mol} / \mathrm{l}$ from the $P_{\mathrm{cr}}$ measured by the Technicon method), ${ }^{9}$ Morris et al used a reaction rate method for $P_{c r}$ measurement, ${ }^{13}$ and Schwartz et al used a Technicon method modified to reduce interference by noncreatinine chromogens. ${ }^{1}$ Therefore, each of the above formulas was calculated twice in all patients - once using the measured $P_{c r}$, and once using the 'corrected' $P_{c r}$, which was calculated by subtraction of $12 \cdot 4 \mu \mathrm{mol} / \mathrm{l}$ from the measured value.

Two methods of statistical analysis were used. First, an Altman-Bland analysis was performed. ${ }^{11}$ The difference between measured and estimated $\log _{e}$ GFR was plotted against the average of the measured and estimated $\log _{e}$ GFR. Further, the ratio geometric mean of measured GFR: geometric mean of estimated GFR was calculated together with $95 \%$ 'reference ranges' for this ratio (the 'limits of agreement' of the measured and estimated GFR). Logarithmic transformation of the data
Table 1 Patient details and GFR results; figures are median (range)

\begin{tabular}{lr}
\hline Age at study (years) & $7 \cdot 1(2 \cdot 2-18 \cdot 5)$ \\
Height $(\mathrm{cm})$ & $118 \cdot 0(88 \cdot 0-177 \cdot 5)$ \\
Plasma creatinine $(\mu \mathrm{mol} / 1)$ & $59(26-202)$ \\
GFR (ml/min $\left./ 1 \cdot 73 \mathrm{~m}^{2}\right)^{\star}$ & \\
Measured from ${ }^{51} \mathrm{Cr}-\mathrm{EDT}$ A plasma & \\
clearance & $87 \cdot 0(37 \cdot 0-211 \cdot 0)$ \\
Estimated (CB-38) & $81 \cdot 9(32 \cdot 9-135 \cdot 2)$ \\
Estimated (CB-40) & $87 \cdot 0(34 \cdot 7-142 \cdot 3)$ \\
Estimated (Schwartz) & $114.9(53 \cdot 6-173 \cdot 0)$
\end{tabular}

${ }^{\star} \mathrm{CB}-38=$ GFR estimated from formula (1); CB-40 = GFR estimated from formula (2); Schwartz=GFR estimated from formula (3); all are calculated using non-corrected plasma creatinine concentration.

was necessary since the spread of the difference between measured and estimated GFR appeared to increase with GFR. Therefore, the limits of agreement are expressed in terms of ratios rather than absolute differences.

Second, the correlation coefficient $(r)$ was calculated, as in previous studies.

\section{Results}

The median (range) height, non-corrected $\mathbf{P}_{\mathrm{cr}}$, measured GFR, and each of the estimated GFRs (using the non-corrected $\mathrm{P}_{\mathrm{cr}}$ ) are shown in table 1.

The Altman-Bland plot of the difference between measured $\log _{e}$ GFR and estimated $\log _{e}$ GFR (estimated from the CounahanBarratt formula, using the non-corrected $\mathrm{P}_{\mathrm{cr}}$ ) against the average of the measured and estimated $\log _{e}$ GFR is shown in fig 1 . The ratio geometric mean of measured GFR: geometric mean of estimated GFR, which is a measure of relative bias (subsequently referred to as 'the bias') and the limits of agreement are shown in table 2. The bias for the Counahan-Barratt formula is 1.12 , implying that on average measured GFRs will be $1 \cdot 12$ times those estimated using the Counahan-Barratt formula. For all but one of the formulas, the bias is significantly different from unity. The bias is even further from unity when the $P_{c r}$ is corrected by subtracting $12.4 \mu \mathrm{mol} / 1$ from the measured value. The only formula in which the bias is not significantly different from unity is

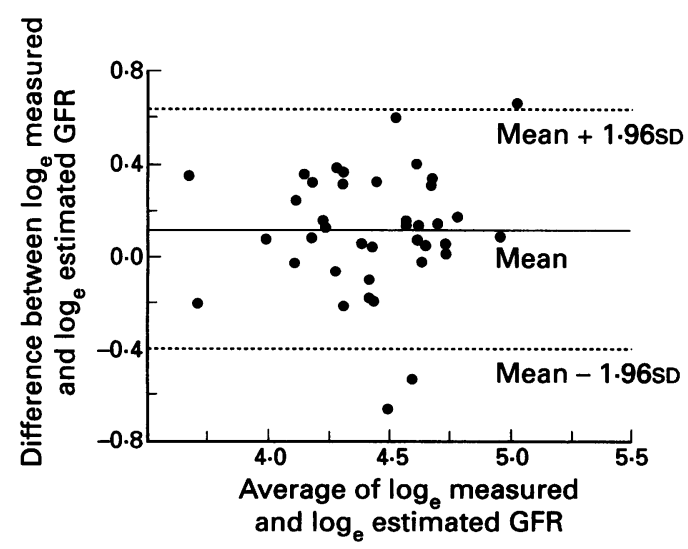

Figure 1 An Altman-Bland plot of the difference between the $\log _{\mathrm{e}}$ GFR measured from the plasma clearance of ${ }^{51} \mathrm{Cr}-E D T A$ and the $\log _{\mathrm{e}}$ GFR estimated from the Counahan-Barratt formula (using the non-corrected plasma creatinine concentration) against the average of the measured and estimated $\log _{\mathrm{e}}$ GFRs. The mean (1.96 SD) value of the difference is shown. 
Table 2 Bias and limits of agreement of measured and estimated GFRs

\begin{tabular}{|c|c|c|c|c|}
\hline & \multicolumn{3}{|l|}{ Bias $^{\star}$} & \multirow[b]{2}{*}{ Limits of agreement $\dagger$} \\
\hline & Mean & $95 \% C I$ & p Value & \\
\hline \multicolumn{5}{|c|}{ Using non-corrected plasma creatinine concentration } \\
\hline Measured GFR $v$ CB-38 & $1 \cdot 12$ & 1.03 to 1.22 & 0.009 & $0 \cdot 67-1.89$ (see fig 1 ) \\
\hline Measured GFR $v$ CB-40 & 1.07 & 0.98 to 1.16 & $0 \cdot 130$ & $0.64-1.79$ \\
\hline Measured GFR $v$ Schwartz & $0 \cdot 84$ & 0.77 to 0.92 & $<0.001$ & $0 \cdot 48-1 \cdot 47$ \\
\hline \multicolumn{5}{|c|}{ Using corrected plasma creatinine concentration } \\
\hline Measured GFR $v$ CB-38 & 0.86 & 0.78 to 0.95 & 0.005 & $0 \cdot 47-1 \cdot 58$ \\
\hline Measured GFR $v$ CB-40 & $0 \cdot 82$ & 0.74 to 0.91 & $<0.001$ & $0 \cdot 45-1 \cdot 51$ \\
\hline Measured GFR $v$ Schwartz & 0.64 & 0.57 to 0.71 & $<0.001$ & $0 \cdot 33-1 \cdot 22$ \\
\hline
\end{tabular}

${ }^{\star}$ Bias $=$ the ratio geometric mean of measured GFR: geometric mean of estimated GFR (see text); $95 \% \mathrm{CI}=95 \%$ confidence interval of the bias; $p=$ probability that the bias is equal to unity. tLimits of agreement $=$ the $95 \%$ reference range for the bias.

the modified Counahan-Barratt formula when the non-corrected $P_{\mathrm{cr}}$ is used.

Furthermore, the limits of agreement are extremely wide in all three formulas, using either the non-corrected or the corrected $\mathbf{P}_{\mathrm{cr}}$. For example, using the Counahan-Barratt formula and non-corrected $P_{c r}$, the measured GFR will be between 0.69 and 1.89 times the estimated value in $95 \%$ of cases; when the corrected $\mathrm{P}_{\mathrm{cr}}$ is used, the equivalent figures are 0.47 and 1.58 .

The correlation coefficients between the measured GFR and each of the estimated GFR values are shown in table 3 , and the scatter plot of measured GFR versus the estimated GFR (from the Counahan-Barratt formula, using the non-corrected $P_{c r}$ ) is shown in fig 2 . Although all the correlation coefficients are highly statistically significant, the figure illustrates the considerable discrepancies between the measured and estimated GFR in many individual patients.

\section{Discussion}

The most important finding of this study is the considerable disagreement between measured and estimated GFR in individual patients. The width of the limits of agreement with measured GFR prevents the use of the Counahan-Barratt and Schwartz formulas in circumstances where accurate determination of the GFR is required for either clinical or research purposes. Use of the corrected (rather than the non-corrected) $\mathrm{P}_{\mathrm{cr}}$ fails to narrow the limits of agreement.

The use of the correlation coefficient is misleading and meaningless in this situation, and the significance value is irrelevant. Moreover, it is apparent from table 3 and fig 2 that a highly significant correlation coefficient may exist despite considerable scatter. The correlation coefficient measures linear association rather than agreement. Two variables may be related without being in agreement; indeed, as Altman and Bland have commented, it would

Table 3 Correlation coefficients between measured GFR and estimated GFR

\begin{tabular}{lcc}
\hline & $r$ & $p$ Value \\
\hline Using non-corrected plasma creatinine concentration \\
Measured GFR $v$ CB-38 & 0.581 & $<0.001$ (see fig 2) \\
Measured GFR $v$ CB-40 & 0.581 & $<0.001$ \\
Measured GFR $v$ Schwartz & 0.524 & 0.001 \\
Using corrected plasma creatinine concentration \\
Measured GFR $v$ CB-38 & 0.550 & $<0.001$ \\
Measured GFR $v$ CB-40 & 0.550 & $<0.001$ \\
Measured GFR $v$ Schwartz & 0.491 & $<0.001$ \\
\hline
\end{tabular}

be surprising if the results obtained from two methods designed to measure the same quantity were not related. ${ }^{11}$ Furthermore, as the value of the correlation coefficient (for this application) depends on both the variation between individuals and that within individuals (that is, measurement error), large interindividual variation relative to measurement error will give a high correlation coefficient, with a highly significant $\mathrm{p}$ value.

The bias is significantly different from unity, except in the case of the modified CounahanBarratt formula using non-corrected $\mathrm{P}_{\mathrm{cr}}$. As expected, use of the corrected $\mathrm{P}_{\mathrm{cr}}$ increases the mean estimated GFR. However, this confers no advantage as the corrected bias is even further from unity than the non-corrected bias with each of the three formulas used.

Data presented by Burghard et al gives a correlation coefficient of 0.91 for 50 subjects, comparing renal clearance of inulin and GFR estimated from the Counahan-Barratt formula. ${ }^{14}$ However, inspection of their figure still shows considerable scatter. The higher value of the correlation coefficient is largely due to the wider range of the measured GFR in their patients. When the data of Burghard et al are analysed in the same way as ours, the results are comparable - although the bias $(0.97$; with $95 \%$ confidence interval 0.89 to $1.05)$ is close to unity, the limits of agreement are just as wide $(0.53-1 \cdot 75)$.

Reference to the initial publications describing the derivation and validation of estimated GFR formulas shows that correlation and regression analyses were used, and that considerable scatter was present despite the high correlation coefficients. ${ }^{910}$ Counahan et al ${ }^{9}$ and other authors have commented on the wide confidence limits for estimated GFRs in individual patients. ${ }^{1516}$ Although it has been suggested that the use of estimated GFR may be appropriate to screen children by identifying those who are very likely to have a normal or an abnormal GFR, ${ }^{13}$ it has also been acknowledged that a renal inulin clearance or radioisotopic plasma clearance method is necessary when an accurate GFR measurement is needed for either clinical or research purposes. ${ }^{14} 16$

In general, inaccuracy of estimated GFR may be due to interindividual differences in

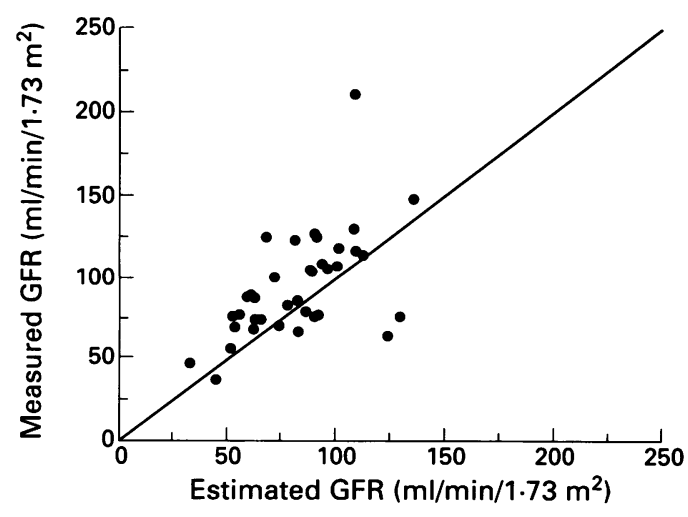

Figure 2 Scatter plot of GFR measured from plasma clearance of ${ }^{51} \mathrm{Cr}$-EDTA against GFR estimated from the Counahan-Barratt formula (using the non-corrected plasma creatinine concentration), with the line of identity. 
body habitus, including reduced muscle bulk in children with cancer, and the use of different methods of $P_{c r}$ measurement. This study comprised an apparently typical group of children who had completed treatment for cancer. None were still malnourished as judged by their weight for height for age, and the estimated GFR of the four overweight patients did not appear to be unrepresentative of the group as a whole. A regression equation between height and $P_{c r}$ derived from one group of children might not be applicable to another group with a different range of body habitus, and the convenience of GFR estimation is lost if validation is required in individual populations before it can be confidently applied.

As there is no clear guidance on desirable levels of accuracy in either clinical or research practice, individual judgment must be exercised for each particular application of a GFR measurement or estimation method. Nevertheless, the results of this study demonstrate that it is not possible to predict accurately the GFR measured from the plasma clearance of ${ }^{51} \mathrm{Cr}$-EDTA in children treated with potential nephrotoxins by using a formula relating height and $\mathbf{P}_{\mathrm{cr}}$. Although the method may be adequate for some clinical purposes (for example, identifying those children in whom GFR is unlikely to be below a certain threshold ${ }^{13}$ ), the limits of agreement shown in table 2 are clearly far too wide to allow confidence in these GFR estimations for either guidance in the clinical decision of whether or not to give further potentially nephrotoxic chemotherapy, or for comparison of the glomerular toxicity of particular treatments.

Furthermore, comparison of GFR measurement methods (including the initial studies of ${ }^{51} \mathrm{Cr}$-EDTA plasma clearance ${ }^{5-8}$ ) has usually been based on the use of correlation and regression analysis, which is not an adequate tool to determine whether two methods of measuring the same variable can be used interchangeably. Reappraisal of currently used methods of GFR measurement in children should include appropriate statistical evaluation.

Dr R Skinner was a MRC Training Fellow. We also thank the Special Trustees of Newcastle Health Authority and the North of England Children's Cancer Research Fund for additional financial support.

1 Schwartz GJ, Brian LP, Spitzer A. The use of plasma creatinine concentration for estimating glomerular filtration rate in infants, children, and adolescents. Pediatr Clin North Am 1987; 34: 571-90.

2 Skinner R, Pearson ADJ, Coulthard MG, et al. Assessmen of chemotherapy-associated nephrotoxicity in children with cancer. Cancer Chemother Pharmacol 1991; 28: 81-92.

3 Shemesh O, Golbetz H, Kriss JP, Myers BD. Limitations of creatinine as a filtration marker in glomerulopathic patients. Kidney Int 1985; 28: 830-8.

4 Chantler C Barratt TM. Estimation of glomerular filtration rate from plasma clearance of 51-chromium edetic acid. Arch Dis Child 1972; 47: 613-7.

5 Brochner-Mortensen J, Giese J, Rossing N. Renal inulin clearance versus total plasma clearance of ${ }^{51} \mathrm{Cr}$-EDTA Clin Lab Invest 1969; 23: 301-5.

6 Chantler C, Garnett ES, Parsons V, Veall N. Glomerula filtration rate measurement in man by the single injection method using ${ }^{51}$ Cr-EDTA. Clin Sci 1969; 37: 169-80.

7 Vorgeli B, Rieduryl H, Donath A, Oetliker O. Comparison of glomerular filtration rate and effective renal plasma flo technique and by means of a standard clearance technique in children. Acta Paediatr Scand 1971; 60: 528-32.

8 Brochner-Mortensen J, Rohbrandt K, Lauritzen RB. Precision of single injection $\left[{ }^{51} \mathrm{Cr}\right.$ ] EDTA plasma clearance and endogenous creatinine clearance determination in children. Scand $\mathcal{F}$ Clin Lab Invest 1977; 37: 625-9.

9 Counahan R, Chantler C, Ghazali S, Kirkwood B, Rose F, Barrett TM. Estimation of glomerular filtration rate from plasma creatinine concentration in children. Arch Dis Child 1976; 51: 875-8.

10 Schwartz GJ, Haycock GB, Edelmann CM Jr, Spitzer A. A simple estimate of glomerular filtration rate in children derived from body length and plasma creatinine. Pediatrics 1976; 58: 259-63.

11 Bland JM, Altman DG. Statistical methods for assessing agreement between two methods of clinical measurement. Lancet 1986; i: 307-10.

12 Black D, James WPT, Besser GM, et al. Obesity. A report of the Royal College of Physicians. $\mathcal{F} R$ Coll Physicians Lond 1983; 17: 5-65.

13 Morris MC, Allanby CW, Toseland P, Haycock GB Chantler C. Evaluation of a height/plasma creatinine formula in the measurement of glomerular filtration rate. Arch Dis Child 1982; 57: 611-4.

14 Burghard R, Offner G, Galaske RG, Brodehl J. Bestimmung der glomerularen filtrationsrate bei kindern aus korperlange und serumkreatinin. Klin Padiatr 1982, 194: 397-9.

15 Davies JG, Taylor CM, White RHR, Marshall T. Clinical limitations of the estimation of glomerular filtration rate from height/plasma creatinine ratio: a comparison with simultaneous ${ }^{51} \mathrm{Cr}$ edetic acid slope clearance. Arch Dis Child 1982; 57: 607-10.

16 Haycock GB. Creatinine, body size and renal function Pediatr Nephrol 1989; 3: 22-4. 